\section{GAST 2014 in Istanbul}

Speziell in Deutschland besteht ein besonderes Verhältnis zur Türkei. Einerseits ist die Türkei seit vielen Jahren beliebtes Urlaubsziel und andererseits bietet sie eine alte, interessante, teils fremde und dadurch spannende Kultur. Viele Menschen, die selbst oder aber deren Eltern aus der Türkei stammen sind heute in Deutschland oder den anderen deutschsprachigen Ländern zu Hause. Ein großer Teil hiervon besitzt auch die entsprechende Staatsbürgerschaft. Neben diesen menschlichen Aspekten entwickelt sich die Türkei auch im Bereich der Wissenschaft und Forschung überproportional schnell. Wohl nicht zuletzt deshalb wurde vom Bundesministerium für Bildung und Forschung am 24.09.2012 eine Richtlinie zur Förderung der verstärkten Zusammenarbeit mit der Türkei veröffentlicht („http://www.bmbf. de/foerderungen/20284.php“).

Innerhalb der Radiologie wurde dies schon früh erkannt, vor allem auch aufgrund schon bestehender sehr enger und guter Kontakte. Nach langen Jahren der Planung eines ersten Meetings in Izmir 2010 kamen nun vom 08.-10. Mai 2014 bereits zum dritten Mal internationale Radiologen zum German-AuStria-Turkey joint venture meeting (GAST) zusammen. Wie schon vor zwei Jahren fand das Meeting auch dieses mal in Istanbul und damit wieder in einer der schönsten Städte der Türkei statt.

Die Kernthemen des diesjährigen Treffens waren „Daily routine work: theory, protocols, basics and pitfalls“, „Research in Radiology“, „Specials (parasites, tuberculosis, obesity)“, „Neuroradiology“, „, Pediatrics“, „Gynaecologic imaging“. Die Vorträ-

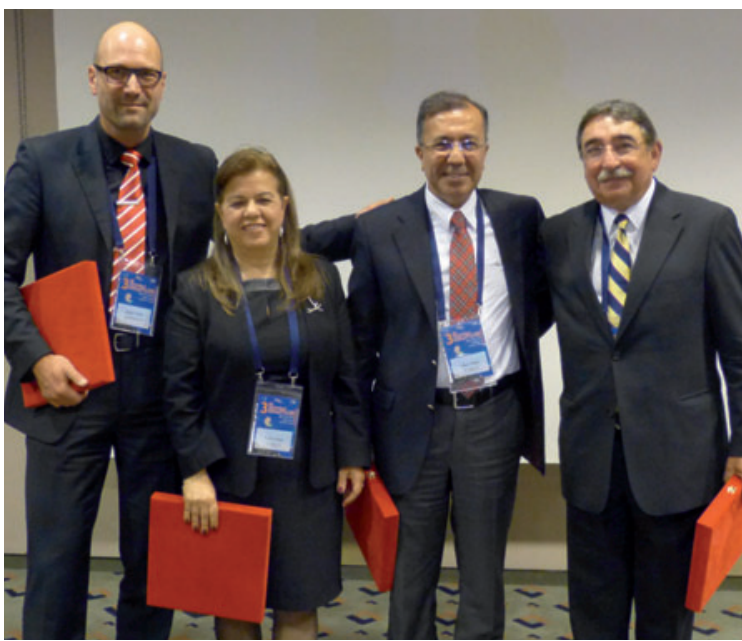

Die stolzen Preisträger des diesjährigen GAST-Awards von links nach rechts: PD Dr. Dr. Stefan Wirth (München), Prof. Nevra Elmas (Izmir), Prof. Ohan Akhan (Ankara) und Prof. Walter Hruby (Wien).

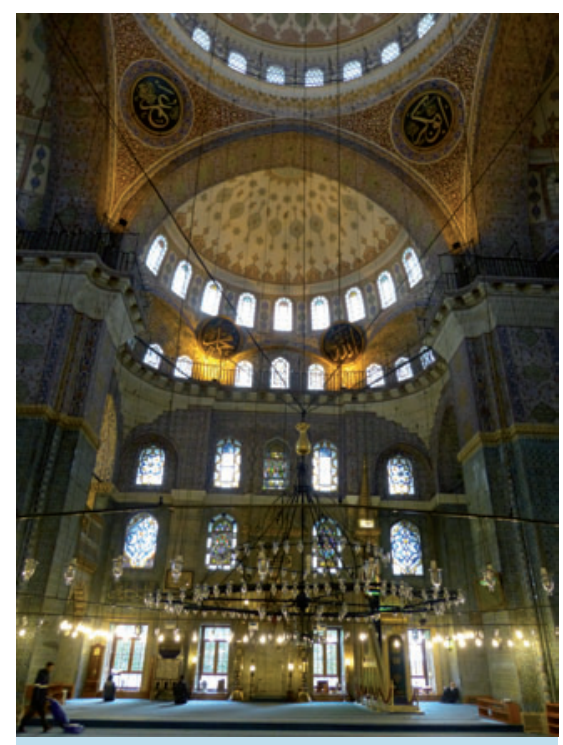

Istanbul als Veranstaltungsort des GAST 2014 ist ein Ort mit allgegenwärtig präsenter Geschichte. An den Gebäuden lassen sich auch heute noch die verschiedenen Epochen erkennen. Hier ein Blick aus der besonders prachtvollen YENI-Moschee

chen) für Ihre besonderen Verdienste um die GAST-Kooperation. Herr Professor Reiser hielt die Laudatio und würdigte besonders die Verdienste und das große Engagement.

Neben dem wissenschaftlichen Programm konnten die Teilnehmer die Stadt Istanbul entdecken, ein magischer Ort der die Kontinente Europa und Asien verbindet. Istanbul ist seit jeher eine Stadt der Toleranz und Brüderlichkeit, wo Moscheen, Kirchen und Synagogen nebeneinander existieren. Die Stadt ist reich an Geschichte, traditionell und gleichzeitig sehr modern, bunt und jung.

Selbstverständlich wurden die Ergebnisse und Erfolge wieder gefeiert und die Freundschaft erneuert. Die türkische Gastfreundschaft ist für Mitteleuropäer kaum zu glauben. So wurde gemeinsam gespeist, getrunken, viel gelacht und die weitere Planung für den nächsten GAST besprochen. Vielleicht sollte das nächste Meeting diesmal im deutschsprachigen Raum stattfinden, um auch den vielen hier tätigen türkischen Kollegen eine Teilnahme zu erleichtern.

Wir verließen die Türkei wieder einmal mit vielen schönen Eindrücken und freuen uns, die doch inzwischen sehr herzliche Verbundenheit mit den türkischen Freunden weiterhin zu pflegen.

PD Dr. Dr. Stefan Wirth 